\title{
Tracking of body size from birth to 7 years of age and factors associated with maintenance of a high body size from birth to 7 years of age - the Norwegian Mother and Child Cohort study $(\mathrm{MoBa})$
}

\author{
Anne Lene Kristiansen ${ }^{1, *}$, Mona Bjelland', Anne Lise Brantsæeter ${ }^{2}$, Margaretha Haugen $^{2}$, \\ Helle Margrete Meltzer ${ }^{2}$, Wenche Nystad ${ }^{2}$ and Lene Frost Andersen ${ }^{1}$ \\ 'Department of Nutrition, Institute of Basic Medical Sciences, University of Oslo, PO Box 1046 Blindern, 0316 Oslo, \\ Norway: ${ }^{2}$ Department of Exposure and Risk Assessment, Division of Environmental Medicine, Norwegian Institute of \\ Public Health, Nydalen, Norway
}

Submitted 14 March 2014: Final revision received 3 September 2014: Accepted 29 September 2014: First published online 10 November 2014

\begin{abstract}
Objective: To examine tracking of body size among children participating in the Norwegian Mother and Child Cohort Study (MoBa) from birth to 7 years of age and additionally to explore child and parental characteristics associated with maintenance of a high body size in this period of life.

Design: Anthropometric data at birth and at 1, 3 and 7 years of age were collected by questionnaires addressed to the mother.

Setting: Participants were recruited from all over Norway during the period 1999-2008.

Subjects: A total of 3771 children had complete anthropometric data at birth and at 1, 3 and 7 years of age; the sample includes children born between 2002 and 2004. Results: Cohen's weighted kappa pointed to fair (0.36) to moderate $(0.43)$ tracking of body size from birth to 7 years of age. Generalized estimating equations further indicated that children in the highest tertile of ponderal index at birth had nearly one unit higher BMI $\left(\mathrm{kg} / \mathrm{m}^{2}\right)$ at the age of 7 years compared with children in other tertiles of ponderal index at birth. Having parents with high BMI $\left(\geq 25 \cdot 0 \mathrm{~kg} / \mathrm{m}^{2}\right)$ increased the odds of having a stable high body size from birth to 7 years of age; moreover, girls had significantly higher odds compared with boys.

Conclusions: The study indicates fair to moderate tracking of body size from birth to 7 years of age. From a public health perspective, early prevention of childhood overweight and obesity seems to be especially important among children of parents having a high BMI.
\end{abstract}

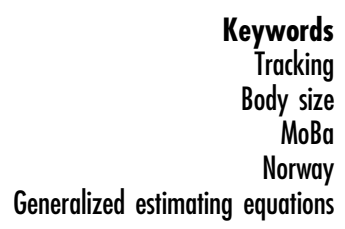

As the prevalence of childhood overweight and obesity has increased worldwide over the past decades ${ }^{(1)}$, the importance of early prevention is highlighted in strategic documents from the $\mathrm{WHO}^{(2)}$ and the European Union ${ }^{(3)}$. Childhood overweight and obesity are important public health challenges because of their associations with both short- and long-term health risks. Short-term health risks include unfavourable psychological consequences like low self-esteem as well as associations with several cardiovascular risk factors ${ }^{(4)}$. Long-term health risks include the maintenance of obesity from childhood into later life ${ }^{(4-6)}$ in addition to increased risk of premature mortality, type 2 diabetes, CVD and morbidity in adulthood ${ }^{(7,8)}$.

Along with the rising prevalence of childhood overweight and obesity, it has become essential to understand not only to what extent such conditions are maintained over time but also the factors influencing this maintenance. Maintenance or stability of overweight or obesity over time is often referred to as 'tracking'. Tracking can be defined as: (i) the relative stability of a certain characteristic over time; or (ii) the predictability of a measurement of a certain risk factor early in life for values of the same risk factor later in life $^{(9)}$. Consequently, the general thought behind tracking is that individuals at risk for diseases later in life can be identified at an early age ${ }^{(10)}$ which possibility can lead to earlier preventive measures or treatment.

Several studies have analysed the association of body size in childhood in relation to body size at subsequent ages. Bayer et al. ${ }^{(11)}$ did a review of studies on BMI tracking. In total forty-eight cohort studies were included, 
mostly from Europe and North America. Age at baseline was divided into four (age groups from less than 10 years of age to 18 years or older) with follow-up time ranging from 0.5 years to 44 years. A high degree of tracking between BMI at baseline and BMI at follow-ups was observed for all age groups, with the highest values seen among the oldest age group (18 years or older $)^{(11)}$. Stocks et $a l .{ }^{(12)}$ reviewed the literature on body size in early childhood (0-4 years of age) and the association with body size at the age of 5-13 years. Of the twenty-one included studies, most were from developed countries in Europe, North America and Australia/New Zealand. The results indicated that large body size in early childhood was related to large body size at subsequent ages. In a recent review by Brisbois et al. ${ }^{(5)}$ analysing the association between childhood obesity at $\leq 5$ years of age and adult ( $\geq 18$ and $\leq 50$ years of age) overweight or obesity, most of the twenty-four included studies found significant associations. Moreover, results pointed to tracking of childhood obesity into adult obesity as early as from 2 years of age.

Lately, there has been an increasing focus on research to identify early risk factors for childhood overweight and obesity. A recent systematic review and meta-analyses reported that several risk factors are already identifiable during the first year of life ${ }^{(13)}$. The review included thirty prospective studies, mostly from North America and Europe. The follow-up times of the studies from birth were at least 2 years and with a maximum of 16 years. Increasing odds of childhood overweight and obesity were found for high birth weight, rapid weight gain in the first year of life, maternal pre-pregnancy overweight and maternal smoking in pregnancy. In addition, a moderate protective effect was found for breast-feeding while no association was found for maternal education at birth.

As illustrated, a considerable number of studies exist on tracking but few consider associations as early as from birth. Moreover, limited data exist for tracking of body size in childhood in the Norwegian population. The objective of the present study was therefore to examine tracking of body size from birth to 7 years of age among children participating in the Norwegian Mother and Child Cohort study (MoBa) and additionally to explore child and parental characteristics associated with maintenance of a high body size from birth to 7 years of age.

\section{Materials and methods}

\section{Participants}

The present study used data from MoBa, a prospective population-based pregnancy cohort study conducted by the Norwegian Institute of Public Health ${ }^{(14)}$. Participants were recruited from all over Norway during the period 1999-2008. The final cohort includes more than 114000 children and $40.6 \%$ of the invited women consented to participate. The present study is based on version 6 of the quality-assured data files released for research in 2011 and includes children born between 2002 and 2004. The study was conducted according to the guidelines laid down in the Declaration of Helsinki and all procedures involving human subjects were approved by the Regional Committee of Medical Research Ethics for South-Eastern Norway. Informed consent was provided by each MoBa participant upon recruitment.

In the present paper we used data from five postal questionnaires addressed to the mother. To be included in the analyses all questionnaires had to be answered. Questionnaire data were available for 101624 participants during pregnancy (QA), 89744 participants at 6 months of age (QB), 66808 participants at 18 months of age (QC), 51447 participants at 36 months of age (QD) and 14181 participants at 7 years of age (QE). A total of 9235 participants responded to all these questionnaires. As pregnant women were recruited into MoBa until December 2008, only 14181 children had reached the age of 7 years and were therefore available for inclusion in the present study.

\section{Definition of overweight/obesity}

Information about weight and length at birth was retrieved from QB. If data were missing from the questionnaire, records from the Medical Birth Registry of Norway ${ }^{(15)}$ were used. Information about weight and length/height at ages 1, 3 and 7 years were retrieved from QC, QD and QE, respectively. Weight was reported in grams and length/ height in centimetres. At the age of 7 years, parents reported their child's weight in kilograms and height in centimetres. To make sure that the anthropometric data corresponded to children at the age of 1,3 and 7 years, only those with data reported close to these ages were included in the present study. Therefore, children 1 year of age correspond to children aged 11-13 months, children 3 years of age correspond to children aged 34-38 months and children 7 years of age correspond to children aged 82-86 months. Ponderal index (PI) was calculated as birth weight in kilograms divided by the cube of birth length in metres $\left(\mathrm{kg} / \mathrm{m}^{3}\right)$. BMI was calculated as weight in kilograms divided by the square of length/height in metres $\left(\mathrm{kg} / \mathrm{m}^{2}\right)$. We chose to use PI as an adiposity index at birth because PI is considered a better measure of adiposity ${ }^{(16)}$ and is a more frequently used weight/length index in neonatal populations than is BMI. We excluded children with unrealistic information on weight and length/height. Tracking of body size from birth to 7 years of age was explored among those 3771 participants with complete anthropometric data at birth and at 1, 3 and 7 years of age.

The age- and gender-specific BMI cut-off points, based on the International Obesity Task Force (IOTF) cut-off points $^{(17)}$, were used to estimate the prevalence of overweight and obesity at the specific age. These cut-off points have recently been proposed by Monasta et al. ${ }^{(18)}$ to be the preferable cut-off points for identification of 
overweight and obesity in childhood as they are defined to pass through BMI of 25.0 and $30.0 \mathrm{~kg} / \mathrm{m}^{2}$, respectively, at the age of 18 years. The term 'overweight including obesity' (OWOB) is used herein to describe the prevalence of overweight and obesity as a total.

\section{Other data}

Questionnaire QB also provided information on parental education levels, paternal height and weight, and maternal pre-pregnancy height and weight. Information on child gender, gestational age, maternal smoking in the third trimester, information about full breast-feeding and age at introduction of solid foods was provided by QB, while breast-feeding during the first 12-14 months of life was provided by QC. Parity and maternal age were retrieved from records from the Medical Birth Registry of Norway ${ }^{(15)}$.

Maternal and paternal educational levels were coded by seven categories and combined into three categories: primary/secondary/comprehensive school, academy/college/ university of $\leq 4$ years and academy/college/university of $>4$ years. In the logistic regression analysis the educational level of the parent with the highest educational level was used and named 'parental educational level'. Self-reported height and weight of the parents were used to calculate their BMI which was further categorized into two groups: normal weight $\left(\mathrm{BMI}<25.0 \mathrm{~kg} / \mathrm{m}^{2}\right)$ and overweight/ obese (BMI $\geq 25 \cdot 0 \mathrm{~kg} / \mathrm{m}^{2}$ ). Gestational age was reported in weeks and categorized into two groups: $<38$ weeks and $\geq 38$ weeks. Maternal smoking status in the third trimester was reported as not smoking, smoking but not every day and smoking every day, then dichotomized into smoking and not smoking. Full breast-feeding at 5 months of age was defined as exclusive breast-feeding and predominant breast-feeding together, hence fully breast-fed infants were those who were either exclusively breast-fed or who received water-based supplementation, but no solid food or formula. Age at introduction of seventeen different types of solid foods was reported by the mother in whole months. The duration of breast-feeding was related to whether or not the child received breast milk at the age of 12-14 months. Five categories of the number of children/ parity were categorized into two groups: first born and not first born. Maternal age, reported as a continuous variable, was categorized into three groups: $\leq 24$ years, 25-34 years and $\geq 35$ years.

\section{Statistical analyses}

Tracking was assessed with Cohen's kappa and generalized estimating equations (GEE). With Cohen's kappa, the agreement between each child's relative positions in rank from birth to 7 years of age was assessed. Kappa values were weighted $\left(\kappa_{\mathrm{w}}\right)$, as disagreement related to distant values is weighted more heavily than disagreement related to more similar values ${ }^{(19)}$. The weighted kappas using weights that were based on the squared distance between the categories were used. In SPSS, the weighted kappa calculation is currently not available; consequently a syntax from the IBM website was used ${ }^{(19)}$. According to Landis and $\mathrm{Koch}^{(20)}$, kappa values of 0 to $0 \cdot 20$ indicate slight agreement, $0 \cdot 21$ to 0.40 fair agreement, 0.41 to 0.60 moderate agreement, 0.61 to 0.80 substantial agreement and 0.81 to 1.00 almost perfect agreement. GEE were used to assess the relationship between all BMI outcomes (i.e. birth, 1, 3 and 7 years of age) and PI at birth, controlling for parental BMI, overweight status at 7 years of age, age at introduction of solid foods, parity and child age. The GEE analysis uses all available data to calculate a single stability coefficient, allowing for adjustment of both timedependent and time-independent covariates. In GEE, we used the unstructured correlation structure as the repeated measurements are assumed to be correlated ${ }^{(21)}$.

Multivariate logistic regression analyses were used to analyse child and parent characteristics associated with a persistent high body size from birth to 7 years of age. A persistent high body size from birth to 7 years of age was defined as including those in the highest tertile of PI at birth ( $n$ 1256), who, at the same time, were overweight/ obese at the age of 7 years.

Results from the univariate analyses (with a criterion of $P<0 \cdot 10)$ and evidence from the literature were used to decide which variables should be examined in the multivariate logistic regression analyses. In the final models, significant variables $(P<0.05)$ were included. All $P$ values are two-sided, and a $5 \%$ level of significance was used. All statistical analyses were performed using the statistical software package $\mathrm{IBM}^{\circledR}$ SPSS $^{\circledR}$ Statistics version $20 \cdot 0$.

\section{Results}

In total, 3771 children had complete information on weight and length at all time points. Table 1 presents selected characteristics of the children and their parents, both in the total sample ( $n$ 3771) and among those in the highest tertile of PI at birth ( $n$ 1256). Most characteristics were equally distributed among the two groups. However, among those in the highest tertile of PI at birth, a slightly lower proportion was first born and a slightly higher proportion was born after 38 weeks of gestation. For girls ( $n$ 1839), mean BMI at birth was 14.1 (SD 1.5 ) kg/m², increasing to 16.8 (SD 1.4$) \mathrm{kg} / \mathrm{m}^{2}$ at 1 year of age, then down to $16.0(\mathrm{sD} 1.5) \mathrm{kg} / \mathrm{m}^{2}$ at 3 years of age and further down to 15.9 (SD 2.0$) \mathrm{kg} / \mathrm{m}^{2}$ at 7 years of age. The corresponding values for boys ( $n$ 1932) were $14 \cdot 2$ (SD 1.5$) \mathrm{kg} / \mathrm{m}^{2}$ at birth, $17.2(\mathrm{SD} 1.4) \mathrm{kg} / \mathrm{m}^{2}$ at 1 year of age, $16.2(\mathrm{SD} 1.6) \mathrm{kg} / \mathrm{m}^{2}$ at 3 years of age and $15.9(\mathrm{sD} 1.8) \mathrm{kg} / \mathrm{m}^{2}$ at 7 years of age. Boys had significantly $(P<0 \cdot 001)$ higher $\mathrm{BMI}$ at ages 1 and 3 years compared with girls. In the total sample, the prevalence of OWOB among boys at 3 and 7 years of age was $12 \cdot 0 \%$ and $11 \cdot 2 \%$, respectively. The corresponding values for girls were $13.2 \%$ and $17 \cdot 0 \%$ (data not shown). 
Table 1 Characteristics of the children and their parents, Norwegian Mother and Child Cohort Study (MoBa)

\begin{tabular}{|c|c|c|c|c|}
\hline & \multicolumn{2}{|c|}{ Total sample* $(n$ 3771) } & \multicolumn{2}{|c|}{ Those in the highest tertile of $\mathrm{PI}$ at birth* $(n 1256)$} \\
\hline & $n$ or mean & $\%$ or SD & $n$ or mean & $\%$ or SD \\
\hline \multicolumn{5}{|l|}{ Gender } \\
\hline Boys & 1932 & 51 & 644 & 51 \\
\hline Girls & 1839 & 49 & 612 & 49 \\
\hline Missing & - & - & - & - \\
\hline \multicolumn{5}{|l|}{ Gestational age } \\
\hline$<38$ weeks & 379 & 10 & 79 & 6 \\
\hline$\geq 38$ weeks & 3308 & 90 & 1150 & 94 \\
\hline Missing & 84 & - & 27 & - \\
\hline \multicolumn{5}{|l|}{ Solid foods at 4 months of age } \\
\hline No & 2000 & 53 & 659 & 53 \\
\hline Yes & 1756 & 47 & 593 & 47 \\
\hline Missing & 15 & - & 4 & - \\
\hline \multicolumn{5}{|l|}{ Full breast-feeding at 5 months of age } \\
\hline Yes & 582 & 16 & 179 & 15 \\
\hline No & 3063 & 84 & 1041 & 85 \\
\hline Missing $(n)$ & 126 & - & 36 & - \\
\hline \multicolumn{5}{|l|}{ Breast-feeding at $12-14$ months of age } \\
\hline Yes & 1424 & 38 & 485 & 39 \\
\hline No & 2347 & 62 & 771 & 61 \\
\hline Missing & - & - & - & - \\
\hline \multicolumn{5}{|l|}{ Parity } \\
\hline First-born child & 1685 & 45 & 482 & 38 \\
\hline Not first-born child & 2080 & 55 & 772 & 62 \\
\hline Missing & 6 & - & 2 & - \\
\hline Maternal age (years) & $30 \cdot 3$ & 4.3 & $30 \cdot 4$ & 4.3 \\
\hline$\leq 24$ & 327 & 9 & 107 & 9 \\
\hline $25-34$ & 2809 & 74 & 919 & 73 \\
\hline$\geq 35$ & 629 & 17 & 228 & 18 \\
\hline Missing & 6 & - & 2 & - \\
\hline \multicolumn{5}{|l|}{ Parental educational level } \\
\hline Primary/secondary/comprehensive school & 1111 & 30 & 387 & 31 \\
\hline Academy/college/university ( $\leq 4$ years) & 1597 & 43 & 546 & 44 \\
\hline Academy/college/university ( $>4$ years) & 1015 & 27 & 304 & 25 \\
\hline Missing $(n)$ & 48 & - & 19 & - \\
\hline \multicolumn{5}{|l|}{ Smoking during pregnancy } \\
\hline Not smoking & 3433 & 93 & 1157 & 94 \\
\hline Smoking & 250 & 7 & 72 & 6 \\
\hline Missing & 88 & - & 27 & - \\
\hline Maternal pre-pregnancy BMI (kg/m²) & $24 \cdot 1$ & $4 \cdot 1$ & $24 \cdot 7$ & $4 \cdot 3$ \\
\hline$<25.0$ & 2489 & 67 & 761 & 62 \\
\hline$\geq 25 \cdot 0$ & 1196 & 33 & 467 & 38 \\
\hline Missing & 86 & - & 28 & _- \\
\hline Paternal BMI $\left(\mathrm{kg} / \mathrm{m}^{2}\right)$ & $25 \cdot 7$ & $3 \cdot 2$ & $25 \cdot 7$ & $3 \cdot 1$ \\
\hline$<25.0$ & 1671 & 46 & 554 & 46 \\
\hline$\geq 25.0$ & 1965 & 54 & 656 & 54 \\
\hline Missing & 135 & - & 46 & - \\
\hline
\end{tabular}

$\mathrm{PI}$, ponderal index.

*Data are presented as numbers and percentages for categorical variables and as means with standard deviations for continuous variables.

For the attrition analyses, participants who had responded to the questionnaire at 7 years of age $(\mathrm{QE})$ were defined as responders ( $n$ 14181). Mean parental BMI, parental educational level and mean maternal age were slightly higher among participants included in the present study ( $n$ 3771) compared with those excluded due to lack of complete information on child anthropometrics ( $n$ 10 410).

Figures 1 and 2 illustrate the development of BMI from birth to 7 years of age, according to tertiles of PI at birth, among boys and girls, respectively. Overall, girls and boys kept their relative position in rank according to body size from birth to 7 years of age. Furthermore, the Cohen's $\kappa_{\mathrm{w}}$ pointed to fair $(0.36)$ to moderate $(0.43)$ tracking of body size from birth to 7 years of age among girls and boys, respectively (Table 2). We also measured the tracking coefficient from birth to 3 years of age, and from 3 to 7 years of age. For both genders, the highest tracking coefficient was seen from 3 to 7 years of age ( 0.58 for boys and 0.55 for girls; data not shown).

The proportion of stability of body size from birth to 7 years of age, based on low, medium and high PI at birth among boys and girls separately, is presented in Table 2 . The largest proportion of stability was seen among boys and girls with medium PI at birth, where about $75 \%$ of boys and girls remained at a medium body size from birth to 7 years of age. Among to those born with a high PI, $13 \%$ 


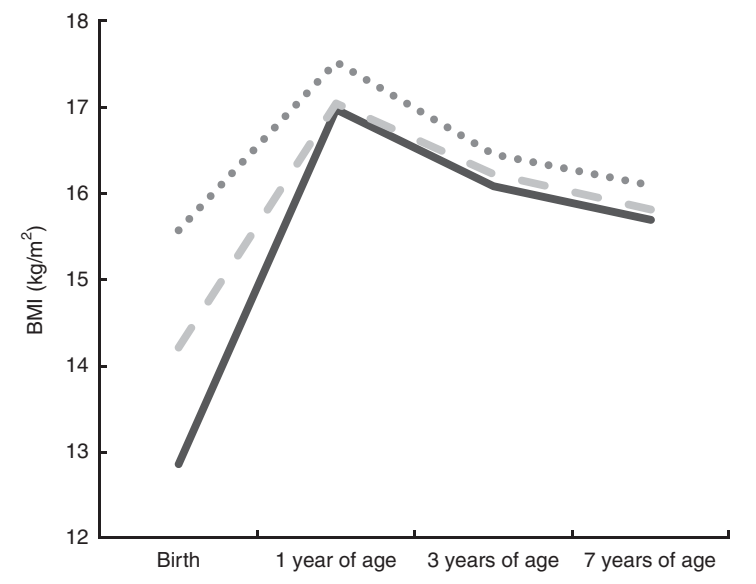

Fig. 1 Tracking patterns of BMI from birth to 7 years of age according to tertiles of ponderal index $(\mathrm{PI})$ at birth $(\longrightarrow$, low $\mathrm{Pl}$ at birth; - - , medium PI at birth; ....., high PI at birth) among boys ( $n$ 1932), Norwegian Mother and Child Cohort Study (MoBa)

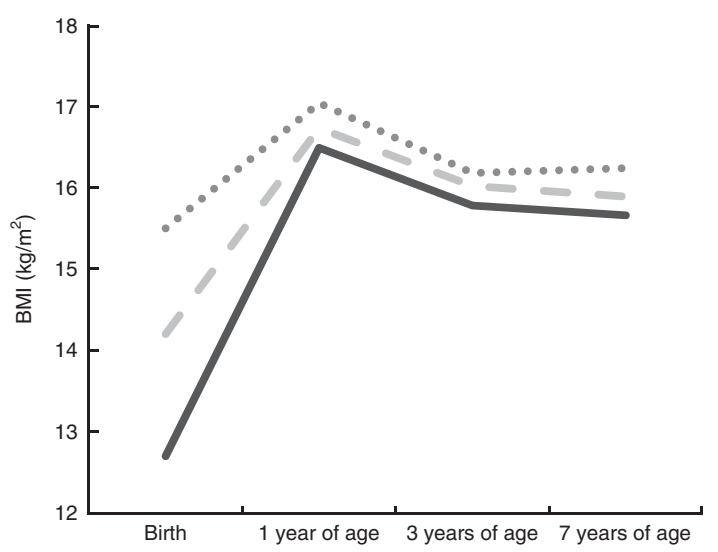

Fig. 2 Tracking patterns of BMI from birth to 7 years of age according to tertiles of ponderal index $(\mathrm{PI})$ at birth $(\longrightarrow$, low $\mathrm{PI}$ at birth; ---, medium $\mathrm{PI}$ at birth; ....., high $\mathrm{PI}$ at birth) among girls (n 1839), Norwegian Mother and Child Cohort Study (MoBa) of the boys and $21 \%$ of the girls remained at a stable high body size from birth to 7 years of age. Moreover, $11 \%$ of the boys and $16 \%$ of the girls with a medium PI at birth increased their body size from birth to 7 years of age (Table 2).

The adjusted GEE analyses indicated that both boys and girls in the highest tertile of PI at birth ( $n$ 1256) had significantly $(P<0.001)$ higher BMI in the period from birth to 7 years of age compared with children in other tertiles of PI at birth ( $n$ 2515). For girls, the value was 0.89 (95\% CI 0.81, $0.98) \mathrm{kg} / \mathrm{m}^{2}$, while the corresponding value for boys was $0.93(95 \%$ CI $0.85,1.00) \mathrm{kg} / \mathrm{m}^{2}$ (data not shown). Consequently, the results can be interpreted as those born with a high PI had nearly one unit higher BMI $\left(\mathrm{kg} / \mathrm{m}^{2}\right)$ at the age of 7 years compared with those not born with a high PI.

Multivariate logistic regression analyses were conducted among those in the highest tertile of PI at birth ( $n$ 1256). Analyses showed that girls had significantly higher odds of having a stable high body size from birth to 7 years of age compared with boys (Table 3). In addition, significant positive associations were seen for maternal prepregnancy BMI and paternal BMI, suggesting that higher parental BMI was associated with higher odds of having a stable high body size in this period of life. Infant feeding practices such as full breast-feeding at 5 months of age, continued breast-feeding beyond 1 year of age and introduction to solid foods before 4 months of age were not associated with tracking of high body size.

When the multivariate logistic regression analyses were analysed with BMI as the measure of birth size rather than PI, the results were more or less in line with those presented in Table 3. However, in the multivariate logistic regression analyses with PI as the measure of birth size, a significant positive association was found for paternal BMI $(P=0 \cdot 045$; Table 3) while with BMI as the measure of birth size, this association was no longer significant $(P=0 \cdot 068)$.

In univariate logistic regression analyses (Table 3) maternal smoking in the third trimester was significantly

Table 2 Stability of body size from birth to 7 years of age and tracking coefficients at 7 years of age $(n 3771)$, Norwegian Mother and Child Cohort Study (MoBa).

\begin{tabular}{|c|c|c|c|c|}
\hline & \multicolumn{3}{|c|}{ Body size measured by BMI at 7 years of age } & \multirow[b]{2}{*}{ Cohen's $K_{\mathrm{w}} \neq$} \\
\hline & Decrease $(\%)^{*}$ & Stable $(\%) \dagger$ & Increase $(\%)^{*}$ & \\
\hline \multicolumn{5}{|l|}{ Boys } \\
\hline \multicolumn{5}{|l|}{$\mathrm{PI}$ at birth } \\
\hline Low (n 644) & nc & $13 \cdot 0$ & $87 \cdot 0$ & \\
\hline Medium ( $n 644)$ & 13.4 & $75 \cdot 3$ & 11.3 & 0.43 \\
\hline High ( $n$ 644) & $87 \cdot 0$ & $13 \cdot 0$ & $\mathrm{nc}$ & \\
\hline \multicolumn{5}{|l|}{ Girls } \\
\hline \multicolumn{5}{|l|}{$\mathrm{Pl}$ at birth } \\
\hline Low (n 613) & $\mathrm{nc}$ & 14.4 & 85.6 & \\
\hline Medium ( $n$ 614) & $9 \cdot 3$ & 74.9 & $15 \cdot 8$ & 0.36 \\
\hline High ( $n$ 612) & 78.8 & $21 \cdot 2$ & $\mathrm{nc}$ & \\
\hline
\end{tabular}

$\mathrm{PI}$, ponderal index; nc, no change as no increase (for those who have high $\mathrm{PI}$ at birth) or decrease (for those who have low $\mathrm{PI}$ at birth) of BMI is possible. ${ }^{*}$ Proportion of children whose relative ranking in body size has decreased or increased from birth to 7 years of age.

†Proportion of children whose relative ranking in body size has not changed from birth to 7 years of age.

$\ddagger$ Tracking coefficient of weighted Cohen's kappa. 
Table 3 Crude and adjusted odds ratios and $95 \%$ confidence intervals of a stable high body size from birth to 7 years of age among those in the highest tertile of PI at birth ( $n$ 1256), Norwegian Mother and Child Cohort Study (MoBa)

\begin{tabular}{|c|c|c|c|c|c|}
\hline & \multirow[b]{2}{*}{$n^{\star}$} & \multicolumn{2}{|c|}{ Crude OR } & \multicolumn{2}{|c|}{ Adjusted OR† } \\
\hline & & OR & $95 \% \mathrm{Cl}$ & OR & $95 \% \mathrm{Cl}$ \\
\hline \multicolumn{6}{|l|}{ Child gender } \\
\hline Boy & 84 & 1.00 & Ref. & 1.00 & Ref. \\
\hline Girl & 130 & 1.80 & $1.33,2.43$ & 1.65 & $1 \cdot 20,2 \cdot 27$ \\
\hline$P$ value & \multicolumn{3}{|c|}{$<0.001$} & \multicolumn{2}{|r|}{$<0.001$} \\
\hline \multicolumn{6}{|l|}{ Solid foods before 4 months of age } \\
\hline No & 110 & 1.00 & Ref. & & \\
\hline Yes & 103 & 1.05 & $0.78,1.41$ & & \\
\hline$P$ value & \multicolumn{3}{|c|}{$0.750^{0.10,1.41}$} & & \\
\hline \multicolumn{6}{|l|}{ Full breast-feeding at 5 months of age } \\
\hline No & 181 & 1.00 & & & \\
\hline Yes & \multirow{2}{*}{\multicolumn{3}{|c|}{$0.49,1.21$}} & & \\
\hline$P$ value & & & & & \\
\hline \multicolumn{4}{|l|}{ Breast-feeding at $12-14$ months of age } & & \\
\hline No & 139 & 1.00 & Ref. & & \\
\hline Yes & 75 & 0.83 & $0.61,1.13$ & & \\
\hline$P$ value & \multicolumn{3}{|c|}{0.240} & & \\
\hline \multicolumn{6}{|l|}{ Smoking in third trimester } \\
\hline Not smoking & 189 & 1.00 & Ref. & 1.00 & Ref. \\
\hline Smoking & 21 & $2 \cdot 11$ & $1.24,3.59$ & 1.60 & $0.89,2.89$ \\
\hline$P$ value & \multicolumn{3}{|c|}{0.006} & \multicolumn{2}{|r|}{0.120} \\
\hline \multicolumn{4}{|l|}{ Parity } & & \\
\hline First-born child & 74 & 1.00 & Ref. & & \\
\hline Not first-born child & 140 & 1.22 & $0.90,1.66$ & & \\
\hline$P$ value & & & & & \\
\hline \multicolumn{4}{|l|}{ Maternal age (years) } & & \\
\hline$\leq 24$ & 23 & 1.00 & Ref. & & \\
\hline $25-34$ & 155 & 0.74 & $0.45,1.21$ & & \\
\hline$\geq 35$ & 36 & 0.69 & $0.38,1.23$ & & \\
\hline $\bar{P}$ trend $\ddagger$ & & & & & \\
\hline \multicolumn{6}{|l|}{ Parental educational level } \\
\hline Primary/secondary/comprehensive school & 77 & 1.00 & Ref. & 1.00 & Ref. \\
\hline Academy/college/university ( $\leq 4$ years) & 94 & 0.84 & $0.60,1.17$ & 0.85 & $0.59,1.22$ \\
\hline Academy/college/university ( $>4$ years) & 38 & 0.58 & $0.38,0.88$ & 0.76 & $0.49,1.19$ \\
\hline$P$ trend $\ddagger$ & & & & \multicolumn{2}{|r|}{0.209} \\
\hline \multicolumn{4}{|l|}{ Maternal pre-pregnancy BMI $\left(\mathrm{kg} / \mathrm{m}^{2}\right)$} & & \\
\hline$<25.0$ & 89 & 1.00 & Ref. & 1.00 & Ref. \\
\hline$\geq 25 \cdot 0$ & 117 & 2.52 & $1 \cdot 86,3.42$ & $2 \cdot 16$ & $1.57,2.97$ \\
\hline $\bar{P}$ value & \multicolumn{3}{|c|}{$<0.001$} & \multicolumn{2}{|r|}{$<0.001$} \\
\hline \multicolumn{4}{|l|}{ Paternal BMI $\left(\mathrm{kg} / \mathrm{m}^{2}\right)$} & & \\
\hline$<25 \cdot 0$ & 75 & 1.00 & Ref. & 1.00 & Ref. \\
\hline$\geq 25.0$ & 129 & 1.56 & $1 \cdot 15,2 \cdot 13$ & 1.40 & $1.00,1.93$ \\
\hline $\bar{P}$ value & & & & & 0.045 \\
\hline
\end{tabular}

PI, ponderal index; Ref., referent category.

${ }^{*}$ Number of children with a stable high body size from birth to 7 years of age within current independent variable.

tOR and $95 \% \mathrm{Cl}$, adjusted for all other variables in the table.

$\ddagger$ Test for linear trend.

associated with a stable high body size from birth to 7 years of age, indicating that smoking was associated with higher odds of having a stable high body size in this period of life. Parental educational level was also a significant variable in univariate analysis, revealing that higher parental education was associated with lower odds of a stable high body size. However, in multivariate analyses these associations were lost.

\section{Discussion}

Findings from the present study indicate moderate tracking of body size from birth to 7 years of age in this group of Norwegian children. Furthermore, children born with a high PI had nearly one unit higher BMI $\left(\mathrm{kg} / \mathrm{m}^{2}\right)$ at the age of 7 years compared with those not born with a high PI. Having parents with high BMI increased the odds of having a stable high body size from birth to 7 years of age; moreover, girls had significantly higher odds compared with boys.

Comparisons of rates of overweight and obesity between countries or even within countries are difficult due to different classifications used to define overweight and obesity and ways of collecting and analysing data ${ }^{(1,22)}$. Nevertheless, two recent papers from Europe indicate that the highest rates of OWOB among pre-school children are observed in countries in the southern regions of Europe ${ }^{(22,23)}$. Like in the 
present work, both papers defined overweight and obesity according to the IOTF criteria ${ }^{(22,23)}$. Among 3-year-olds, the range in European countries was reported to be from $7 \%$ in Belgium to $30 \%$ in Spain, with data collected in the period from 1998 to $2007^{(22)}$. The overall prevalence of OWOB in our study was $13 \%$ among the 3 -year-olds and $14 \%$ among the 7-year-olds. Similar findings, using IOTF criteria, have been observed in other Norwegian studies. In the Bergen Growth Study $^{(24)}$ conducted in 2003-2006, the OWOB prevalence among 3- and 7-year-olds was $11 \%$ and $18 \%$, respectively. Further, in a survey of 1774 children born in 1999 and 2000 in the county of Finnmark ${ }^{(25)}$, the OWOB prevalence among 6-year-olds was 19\%. Lastly, in a national representative sample of 3166 Norwegian children being 8 years in 2010, the overall prevalence of OWOB was $19 \%{ }^{(26)}$. A common finding in the present study, in earlier Norwegian studies $^{(24-26)}$ and in the European studies ${ }^{(22,23)}$ is the higher prevalence of OWOB among girls compared with boys. This warrants discussion about whether this gender difference truly exists or if it is related to the classification criteria used in the IOTF cut-off ${ }^{(24)}$.

In the present study we used Cohen's $\kappa_{\mathrm{w}}$ to assess tracking. One advantage of Cohen's $\kappa_{\mathrm{w}}$ is that length of movements is taken into account when the magnitude of tracking is measured ${ }^{(27)}$. However, if we assessed the correlation between BMI values from birth to 7 years of age with Pearson's correlation coefficient, BMI at 7 years was significantly $(P<0.001)$ correlated with BMI at birth $(r=0 \cdot 16)$, at 1 year of age $(r=0 \cdot 39)$ and at 3 years of age $(r=0.43)$. The tracking values identified in our study are somewhat lower than those reported among children in the age group of less than 10 years at baseline included in the review by Bayer et al. ${ }^{(11)}$. In that review, the correlation between BMI at baseline and BMI at 5- to 10-year follow-up was 0.74 and 0.67 , respectively. However only very few studies included in that review examined tracking of body size from as early as the first year of life. One that did so was an old cohort study from Finland ${ }^{(28)}$ in which tracking of BMI was assessed with Pearson's correlation coefficient. That Finnish study included 100 children born in 1981-1982 and data on their BMI were available when the children were 6 months, 7 and 15 years old. BMI at 6 months of age was significantly correlated with BMI at 7 years of age $(r=0.32)$, but not with BMI at 15 years of age $(r=0 \cdot 11)$. Moreover, BMI at 7 years of age was significantly correlated with BMI at 15 years of age $(r=0 \cdot 69)$. Another study included in the review by Bayer et $a l .{ }^{(11)}$ with tracking data from the first year of life was a Dutch cohort study published in $2006^{(29)}$. Included in the study were 105 children born between 1990 and 1993, with tracking of BMI from birth to 12 years of age assessed with Pearson's correlation coefficient. Data on BMI were available at birth, at 6 months and at 1, 2, 3, 4, 7 and 12 years of age. From 1 to 7 years of age, BMI were significantly correlated with BMI at 12 years of age with correlations increasing from 0.24 (at 1 year of age) to 0.76 (at 7 years of age). As in the Finnish and the Dutch studies $^{(28,29)}$, we also observed higher tracking values with increasing age of the participants. This also corresponds to observations of the other studies included in the review by Bayer et $a l .{ }^{(11)}$. Even so, a recently published cohort study from the Netherlands ${ }^{(30)}$, where 762 participants' height and weight were measured on average twenty-one times in the period from birth to 18 years of age, reported that the age interval 2-6 years was the earliest and most critical growth period for predicting adult overweight.

To our knowledge few authors have assessed tracking of body size with use of GEE. One of the strengths of this method is that all available data on body size are used to calculate a single stability coefficient, allowing for adjustment of covariates. In our analyses we observed that those born with a high PI had nearly one unit higher BMI $\left(\mathrm{kg} / \mathrm{m}^{2}\right)$ at the age of 7 years compared with those not born with a high PI. For girls, the value was $0.89 \mathrm{~kg} / \mathrm{m}^{2}$ while the corresponding value for boys was $0.93 \mathrm{~kg} / \mathrm{m}^{2}$. In a recently published paper Pearson et al. ${ }^{(31)}$ examined tracking of Australian children's BMI. In that study, data were collected for 175 children in 2002-2003 when the children were 5-6 years of age, while the follow-up data were collected in 2006 and 2008. The GEE analyses pointed to a high degree of tracking of BMI units, with values of 0.74 for boys and 0.78 for girls. Hence, BMI was found to be highly stable and followed by increase in BMI units in this period of life ${ }^{(31)}$.

It is suggested that genes have an important effect on BMI variation, but the effect of the common shared environment is also substantial in childhood ${ }^{(32)}$. Several recent reviews have suggested that parental obesity may be an important risk factor for obesity later in life and it seems like maternal obesity is especially important ${ }^{5,13,33,34)}$. This is also in line with findings in the present study, where the odds of having a stable high body size from birth to 7 years of age was more than doubled among children of mothers with a prepregnancy BMI of $\geq 25.0 \mathrm{~kg} / \mathrm{m}^{2}$ compared with children of mothers with a pre-pregnancy BMI of $<25.0 \mathrm{~kg} / \mathrm{m}^{2}$. The same was observed according to paternal BMI, but the association was not as strong as seen for maternal prepregnancy BMI. We further observed that girls had higher odds of having a stable high body size from birth to 7 years of age compared with boys. This might be due to the fact that girls tend to have higher prevalence of OWOB in childhood than boys ${ }^{(22-26)}$, as discussed earlier. Infant feeding practices have been associated with development of childhood overweight and obesity. Previous reviews have reported that a history of breast-feeding is associated with a reduced risk of overweight and obesity later in life ${ }^{(13,35,36)}$, while this was not observed for adult obesity ${ }^{(5)}$. Further, Weng et $a l .{ }^{(13)}$ reported in their review that 'there was some evidence supporting the early introduction of solid foods as a risk factor for later overweight'. In our analyses, full breastfeeding at 5 months of age, breast-feeding at 12-14 months of age and introduction to solid foods before 4 months of 
age were not significantly associated with having a stable high body size from birth to 7 years of age. Maternal smoking in pregnancy has been found to be a strong risk factor for childhood ${ }^{(13)}$ and adult ${ }^{(5)}$ overweight and obesity. A meta-analysis including seven studies $^{(13)}$ showed that children of smoking mothers had increased risk of becoming overweight compared with children of non-smoking mothers. In univariate logistic regression analysis in the present study, maternal smoking in the third trimester was significantly associated with an increased risk of having a stable high body size from birth to 7 years of age. However, in multivariate analyses this association was no longer significant. In the present study, increasing parental education was significantly associated with lower odds of a stable high body size from birth to 7 years of age in univariate analysis, but this association was lost in multivariate analyses. This is in line with the review by Weng et $a l .^{(13)}$ where no association with childhood overweight was found for maternal education at birth, but in contrast to the findings of Shrewsbury and Wardle in their systematic review ${ }^{(37)}$ where the level of parental education was inversely associated with adiposity in fifteen out of twenty studies.

\section{Strengths and limitations}

Strengths of our study are the large sample size and the relatively long follow-up time, including data on important infant feeding practices and characteristics of the children and their parents. However, to be included in the present study, the participants had to have reported data from several questionnaires and this might have led to the inclusion of the most interested participants, possibly reducing the generalizability of the results.

Our results showed that most children kept their relative position in rank over time, when grouped by PI at birth. By introducing cut-offs on continuous variables, it is however important to notice that some information will be lost when outcome variables are grouped ${ }^{(21)}$. Moreover, it is also important to be aware that individuals in the extreme groups, such as those in the highest tertile of PI at birth, can move in only one direction (to less extreme values) while those in the middle group can move in both directions. Therefore, a tendency towards regression to the mean can be observed as participants with extreme values tend to have less extreme values in follow-up measurement(s).

Weight and length/height of the children were reported by the parents. As health-care personnel measure children's weight and length/height at hospitals at birth and at child health clinics at 1 and 3 years of age in Norway, data from the child's health card might have been referred to when such data were reported. On the other hand, if the health card was not used, we do not know whether the parents estimated the child's weight and length/height or if they actually measured it. In a recent paper by Huybrechts et $a l{ }^{(38)}$ it was concluded that at a group level, there were no differences in accuracy with regard to parent-measured or parent-estimated height, weight and BMI of Belgian children 3-7 years of age. Still, parent-measured values gave higher correlations with nurse measurements compared with parent-estimated values. Nevertheless, the OWOB prevalences presented in the current paper are comparable to recently published Norwegian OWOB prevalences in the same age groups ${ }^{(24-26)}$.

Children included in our study were born between 2002 and 2004. In this period, the questionnaire to be answered by the father during pregnancy did not include questions about paternal height and weight. As a consequence of this, paternal height and weight were reported by the mother in QA. However, paternal height and weight were included in the questionnaire to the fathers from late 2005, and a total of 32867 participants in the entire MoBa cohort had information about paternal height and weight reported by both mothers and fathers. The Spearman correlation between maternal and paternal reporting of paternal weight and height was 0.99 and 0.98 , respectively. Hence, paternal data reported by the mothers seem to be precise.

Attrition analyses showed that there were significant differences in selected characteristics of the participants included in the analyses compared with those excluded. Still, at the absolute level there were minor differences between the two groups except that those included tended to have higher educational level than those excluded. Hence, it is assumed that these differences have likely not influenced our findings in any major way. The low participation and loss to follow-up in MoBa in general may be of concern. Women participating in MoBa include more women with higher educational level and more nonsmokers than the general population of pregnant women. However, evaluation of this non-representativeness in MoBa showed that it did not affect exposure-outcome associations $^{(39,40)}$. Mean maternal pre-pregnancy BMI in the total sample of responders to QA was more or less the same as that of our study participants $\left(24.0 \mathrm{~kg} / \mathrm{m}^{2}\right.$ among $n 97123 v .24 \cdot 1 \mathrm{~kg} / \mathrm{m}^{2}$ among $n 3771$ ). The same was seen with regard to paternal BMI reported by the mother in QA and our study participants $\left(25.8 \mathrm{~kg} / \mathrm{m}^{2}\right.$ among $n 96976 v$. $25.7 \mathrm{~kg} / \mathrm{m}^{2}$ among $n$ 3771). Thus, it seems like data on parental BMI in our study correspond to such data in the total cohort. Further, parental BMI was based on self-reported rather than measured height and weight; hence, overweight/obesity prevalence could have been under-reported. In addition, height and weight data were collected during pregnancy of the participating child and as overweight/obesity tends to increase with age, the data could have been further under-reported.

\section{Conclusion}

Findings from earlier studies have suggested tracking of childhood obesity into subsequent ages; we have extended this knowledge further by presenting Norwegian 
data indicating moderate tracking of body size from birth to 7 years of age. Consequently, in a public health perspective where it is of importance to prevent childhood overweight and obesity, the early prevention seems to be especially important among children of parents having a high BMI.

\section{Acknowledgements}

Financial support: The Norwegian Mother and Child Cohort Study (MoBa) is supported by the Norwegian Ministry of Health and the Ministry of Education and Research, the National Institutes of Health/National Institute of Environmental Health Sciences (contract number N01-ES-75558), the National Institutes of Health/National Institute of Neurological Disorders and Stroke (grant numbers UO1 NS 047537-01 and UO1 NS 047537-06A1) and the Norwegian Research Council/Functional Genomics (FUGE) (grant number 151918/S10). The funders had no role in the design, analysis or writing of this article. Conflict of interest: None. Authorship: A.L.K. conducted the statistical analyses and wrote the manuscript in close collaboration with L.F.A. M.B., A.L.B., M.H., H.M.M. and W.N. participated in designing the study and/or in the final editing of the manuscript. All authors have critically revised the manuscript and read and approved the final version. Ethics of buman subject participation: The study was conducted according to the guidelines laid down in the Declaration of Helsinki and all procedures involving human subjects were approved by the Regional Committee of Medical Research Ethics for South-Eastern Norway.

\section{References}

1. Wang Y \& Lobstein T (2006) Worldwide trends in childhood overweight and obesity. Int J Pediatr Obes 1, 11-25.

2. World Health Organization (2008) Prevention and Control of Non-Communicable Diseases: Implementation of the Global Strategy. 61st World Health Assembly, WHA 11.5. Geneva: WHO.

3. European Commission (2005) The EU Platform for Action on Diet, Physical Activity and Health. Brussels: European Commission, Directorate-General Health \& Consumers.

4. Reilly JJ, Methven E, McDowell ZC et al. (2003) Health consequences of obesity. Arch Dis Child 88, 748-752.

5. Brisbois TD, Farmer AP \& McCargar LJ (2012) Early markers of adult obesity: a review. Obes Rev 13, 347-367.

6. Singh AS, Mulder C, Twisk JW et al. (2008) Tracking of childhood overweight into adulthood: a systematic review of the literature. Obes Rev 9, 474-488.

7. Park MH, Falconer C, Viner RM et al. (2012) The impact of childhood obesity on morbidity and mortality in adulthood: a systematic review. Obes Rev 13, 985-1000.

8. Reilly JJ \& Kelly J (2011) Long-term impact of overweight and obesity in childhood and adolescence on morbidity and premature mortality in adulthood: systematic review. Int $J$ Obes (Lond) 35, 891-898.

9. Twisk JW, Kemper HC \& Mellenbergh GJ (1994) Mathematical and analytical aspects of tracking. Epidemiol Rev 16, 165-183.
10. Twisk JW (2003) The problem of evaluating the magnitude of tracking coefficients. Eur J Epidemiol 18, 1025-1026.

11. Bayer O, Kruger H, von Kries R et al. (2011) Factors associated with tracking of BMI: a meta-regression analysis on BMI tracking. Obesity (Silver Spring) 19, 1069-1076.

12. Stocks T, Renders CM, Bulk-Bunschoten AM et al. (2011) Body size and growth in 0- to 4-year-old children and the relation to body size in primary school age. Obes Rev 12, 637-652.

13. Weng SF, Redsell SA, Swift JA et al. (2012) Systematic review and meta-analyses of risk factors for childhood overweight identifiable during infancy. Arch Dis Child $\mathbf{9 7}$, 1019-1026.

14. Magnus P, Irgens LM, Haug K et al. (2006) Cohort profile: the Norwegian Mother and Child Cohort Study (MoBa). Int J Epidemiol 35, 1146-1150.

15. Irgens LM (2000) The Medical Birth Registry of Norway. Epidemiological research and surveillance throughout 30 years. Acta Obstet Gynecol Scand 79, 435-439.

16. Howe LD, Tilling K, Benfield L et al. (2010) Changes in ponderal index and body mass index across childhood and their associations with fat mass and cardiovascular risk factors at age. PLoS One 5, e15186.

17. Cole TJ, Bellizzi MC, Flegal KM et al. (2000) Establishing a standard definition for child overweight and obesity worldwide: international survey. BMJ 320, 1240-1243.

18. Monasta L, Lobstein T, Cole TJ et al. (2011) Defining overweight and obesity in pre-school children: IOTF reference or WHO standard? Obes Rev 12, 295-300.

19. IBM Corporation (date not known) Weighted Kappa syntax for SPSS. http://www-01.ibm.com/support/docview.wss?uid= swg21477357 (accessed March 2013).

20. Landis JR \& Koch GG (1977) The measurement of observer agreement for categorical data. Biometrics 33, 159-174.

21. Twisk J (2003) Applied Longitudinal Data Analysis for Epidemiology - A Practical Guide, 1st ed. New York: Cambridge University Press.

22. Cattaneo A, Monasta L, Stamatakis E et al. (2010) Overweight and obesity in infants and pre-school children in the European Union: a review of existing data. Obes Rev 11, 389-398.

23. van Stralen MM, te Velde SJ, van Nassau F et al. (2012) Weight status of European preschool children and associations with family demographics and energy balance-related behaviours: a pooled analysis of six European studies. Obes Rev 13, Suppl. 1, 29-41.

24. Juliusson PB, Eide GE, Roelants M et al. (2010) Overweight and obesity in Norwegian children: prevalence and sociodemographic risk factors. Acta Paediatr 99, 900-905.

25. Kokkvoll A, Jeppesen E, Juliusson PB et al. (2012) High prevalence of overweight and obesity among 6-year-old children in Finnmark County, North Norway. Acta Paediatr 101, 924-928.

26. Biehl A, Hovengen R, Groholt EK et al. (2013) Adiposity among children in Norway by urbanity and maternal education: a nationally representative study. BMC Public Health 13, 842 .

27. Cohen J (1968) Weighted kappa: nominal scale agreement with provision for scaled disagreement or partial credit. Psychol Bull 70, 213-220.

28. Fuentes RM, Notkola IL, Shemeikka S et al. (2003) Tracking of body mass index during childhood: a 15-year prospective population-based family study in eastern Finland. Int J Obes Relat Metab Disord 27, 716-721.

29. Vogels N, Posthumus DL, Mariman EC et al. (2006) Determinants of overweight in a cohort of Dutch children. $\mathrm{Am} \mathrm{J}$ Clin Nutr 84, 717-724.

30. De Kroon ML, Renders CM, Van Wouwe JP et al. (2010) The Terneuzen birth cohort: BMI changes between 2 and 6 years correlate strongest with adult overweight. PLoS One 5, e9155. 
31. Pearson N, Salmon J, Campbell K et al. (2011) Tracking of children's body-mass index, television viewing and dietary intake over five-years. Prev Med 53, 268-270.

32. Silventoinen K, Rokholm B, Kaprio J et al. (2010) The genetic and environmental influences on childhood obesity: a systematic review of twin and adoption studies. Int J Obes (Lond) 34, 29-40.

33. Patro B, Liber A, Zalewski B et al. (2013) Maternal and paternal body mass index and offspring obesity: a systematic review. Ann Nutr Metab 63, 32-41.

34. Ruager-Martin R, Hyde MJ \& Modi N (2010) Maternal obesity and infant outcomes. Early Hum Dev 86, 715-722.

35. Duijts L, Ramadhani MK \& Moll HA (2009) Breastfeeding protects against infectious diseases during infancy in industrialized countries. A systematic review. Matern Child Nutr 5, 199-210.

36. Ip S, Chung M, Raman G et al. (2007) Breastfeeding and Maternal and Infant Health Outcomes in Developed
Countries. Evidence Report/Technololgy Assessment no. 153. Rockville, MD: Agency for Healthcare Research and Quality, US Department of Health and Human Services.

37. Shrewsbury V \& Wardle J (2008) Socioeconomic status and adiposity in childhood: a systematic review of crosssectional studies 1990-2005. Obesity (Silver Spring) 16, $275-284$.

38. Huybrechts I, Himes JH, Ottevaere C et al. (2011) Validity of parent-reported weight and height of preschool children measured at home or estimated without home measurement: a validation study. BMC Pediatr 11, 63.

39. Nilsen RM, Suren P, Gunnes N et al. (2013) Analysis of self-selection bias in a population-based cohort study of autism spectrum disorders. Paediatr Perinat Epidemiol 27, 553-563.

40. Nilsen RM, Vollset SE, Gjessing HK et al. (2009) Selfselection and bias in a large prospective pregnancy cohort in Norway. Paediatr Perinat Epidemiol 23, 597-608. 